\title{
Nalidixic acid resistant Salmonella paratyphi $A$ causing urinary tract infection in a patient with nephrolithiasis: case report
}

\author{
Sarika Jain, Shamma Arora, Rumpa Saha, Iqbal R. Kaur \\ Department of Microbiology, U.C.M.S \& G.T.B.Hospital, Dilshad garden, Delhi, India-110095
}

\section{SUMMARY}

Salmonella urinary tract infections (UTIs) are unusual and occur most often in infants and those over 60 years. S. paratyphi has been reported extremely rarely as a cause of UTI. Recovery of $S$. typhi is also rare from urine and can occur following a recent episode of typhoid fever, or in chronic carrier states involving the urinary system, and occasionally following localized UTI due to S. typhi. Studies have reported $0.07 \%$ of urinary tract infections diagnosed in 15 year duration to be due to nontyphoidal salmonella (NTS) and $0.24 \%$ of organisms cultured from urine were NTS. NTS species isolated from urine include $S$. enteritidis, S. typhimurium, S. heidelberg, S. infantis, S. newport. Cases of other unusual extra-intestinal infections caused by $S$. paratyphi $A$ include fulminant hepatic failure, primary septic arthritis of the hip and pleural effusion. Because of its extreme rarity as a uropathogen, and importance of administering appropriate antimicrobial for treating urinary tract infection caused by nalidixic acid resistant and fluoroquinolone sensitive Salmonella paratyphi $A$, we report such an infection from India in an elderly man with renal and ureteric stones.

Key words: Nalidixic acid resistance, Salmonella paratyphi A, urinary tract infection.

\section{CASE REPORT}

A sixty eight year old man presented in surgery OPD with fever and right sided intermittent loin pain for two months. He also complained of dysuria while there was no history of increased urinary frequency or urgency. His previous records revealed that he had suffered from recurrent attacks of UTI due to Proteus mirabilis in the past. He was prescribed ciprofloxacin but had taken incomplete treatment. The patient did not have history of diabetes mellitus, any chronic ailment such as tuberculosis or immunosuppressive state or hospitalisation. No past history of typhoid, paratyphoid or any other febrile illness was present. On general examination, the patient was febrile while the other vital signs were normal. He did

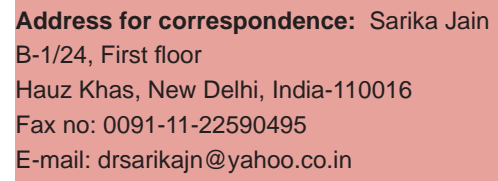

DOI: 10.5530/ijmedph.4.2011.9 not have pallor or jaundice. On abdominal examination, right sided flank tenderness was present. No mass or tenderness was felt in right or left hypochondriac region. Laboratory investigations showed that leucocyte count was $10,000 / \mathrm{mm}^{3}$ with slight lymphocytosis $(55 \%$ of total). Liver and kidney function tests were normal. Ultrasound of abdomen revealed multiple renal and uretric calculi. On urinalysis, pus cells, red blood cells and triple phosphate crystals were seen. No egg/microfilaria was seen. On culture of urine on CLED media, pure growth of non-lactose fermenting colonies were isolated in significant counts $(>1,00,000 \mathrm{CFU} / \mathrm{mL})$. Biochemically, Salmonella Paratyphi $A$ was suspected and identified by API (Bio, Merieux SA, France). The isolate was further confirmed serologically (O2, H:a positive). The organism was sensitive to chloramphenicol $(30 \mu \mathrm{g})$, nitrofurantoin $(300 \mu \mathrm{g})$, ampicillin $(10 \mu \mathrm{g})$, amoxycillin+clavulanic acid $(20 \mu \mathrm{g}+10 \mu \mathrm{g})$, norfloxacin $(300 \mu \mathrm{g})$, ciprofloxacin $(5 \mu \mathrm{g})$, gentamicin $(10 \mu \mathrm{g})$, amikacin $(30 \mu \mathrm{g})$, piperacillintazobactam $(100 / 10 \mu \mathrm{g})$, ceftriaxone $(30 \mu \mathrm{g})$, cefotaxime $(75 \mu \mathrm{g})$ and imipenem $(10 \mu \mathrm{g})$, but was resistant to nalidixic acid $(30 \mu \mathrm{g})$. Blood culture was sterile and no pathogenic organism was isolated on stool culture. The patient was 
started on intravenous ceftriaxone. The repeat urine culture after one week was sterile.

\section{DISCUSSION}

E. coli is predominant cause of UTI almost exclusively followed by Proteus mirabilis, enterococci, Klebsiella, and Staphylococcus aureus. However, only $5 \%$ of the UTI are caused by remaining micro-organisms such as strains of alcaligenes group, Citrobacter, Sarcina, Neisseria, and also Shigella and Salmonella, although the latter two are extremely seldom reported. Urinary tract manifestations in salmonella infections occur in five clinical forms: transient bacilliuria (following a recent typhoid fever as a part of natural history of disease or in chronic carrier status), perinephric abscess, pyelonephritis, nephrolithiasis and cystitis. Salmonella UTI may occur in conjunction with gastroenteritis or as an isolated event. Studies have analysed UTI due to NTS and observed it predominantly in elderly patients with underlying diseases, especially diabetes mellitus, urologic abnormalities such as nephrolithiasis, hydronephrosis, anatomic abnormalities, schistosomiasis, tuberculosis and neoplasms of the kidney and immunosuppression ${ }^{1}$ and S. enteritidis was the most common serotype followed by S. typhimurium ${ }^{2}$. UTI due to $S$. paratyphi has been reported extremely rarely and that too resistant to nalidixic acid. While a significant number of Salmonella-associated UTIs are linked to persons with one or more co-morbid conditions, many cases of bacteriuria occur in individuals without known risk factors ${ }^{3}$. Besides, salmonella UTI has not been found to differ clinically from other members of the Enterobacteriaceae; only in serious co-morbid conditions such as renal transplant recipients, was the course more serious ${ }^{4}$.

In our case, we isolated $S$. paratyphi $A$ on urine culture of an elderly OPD patient with history of recurrent episodes of UTI with nephrolithiasis and ureteric stone. As the patient had no history of past hospital admissions, the isolate was community acquired and sensitive to all tested antibiotics except nalidixic acid. Since blood culture was sterile at the time of presentation, Salmonella bacteriuria was perhaps either due to local infection of the urinary tract or because of previous unnoticed, asymptomatic blood infection. The urinary stone may have formed due to inadequately treated and recurrent, chronic infection by Proteus mirabilis and may have acted as a nidus of infection for Salmonella paratyphi A. The presence of proteinuria, haematuria, pyuria, magnesium phosphate crystals, isolation of $S$. paratyphi $A$ in pure growth and in significant counts $(>1,00,000 \mathrm{CFU} / \mathrm{mL})$ suggests local infection rather than colonization or fecal contamination. Few cases of UTI due to Salmonella paratyphi $A$ have been reported in literature till date. Fawzia \& Al-Otaibi have reported Salmonella paratyphi $A$ in urine of a middle aged man with nephrolithiasis and hydronephrosis where urinary schistosomiasis was presumably the underlying predisposing condition ${ }^{5}$.

Another noteworthy point is that our isolate was nalidixic acid resistant and according to CLSI recommendations, in Salmonella extra-intestinal infections, if the isolate is fluoroquinolone sensitive and nalidixic acid resistant in vitro, then antibiotics other than fluoroquinolone should be prescribed to patients because the patient will not respond to fluoroquinolones and that might be the reason for treatment failure.

In conclusion, the present case may be the acute presentation of the chronic Salmonella infection of the calculi as the presence of stones, which may harbour organisms often, leads to multiple relapses or to the development of chronic urinary carrier. Since localised infection of urinary tract due to salmonella tends to be chronic and persistent, therefore, besides surgical removal of stones, vigorous, prolonged and judicious treatment with alternative antibiotics such as azithromycin or ceftriaxone is of paramount importance in eradicating infective foci in fluoroquinolone sensitive and nalidixic acid resistant isolates leading to such infections

\section{REFERENCES}

1. Tena D, Gonzalez-Praetorius A, Bisquert J. Urinary tract infection due to non-typhoidal Salmonella: Report of 19 cases. Journal of Infection, 2007;54(3):245-249.

2. Ramos JM, Aguado JM, Garcia-Corbeira P, Alés JM, Soriano F. Clinical spectrum of urinary tract infections due to non-typhoidal Salmonella species. Clin Infect Dis, 1996;23:388-390.

3. Paterson DL, Harrison MW, Robson JMB. Clinical spectrum of urinary tract infections due to nontyphoidal Salmonella species. Clin Infect Dis, 1997;25:754.

4. Allerberger FJ, Dierich MP, Ebner A, Keating MR, Steckelberg JM, Yu PK. Urinary tract infection caused by nontyphoidal Salmonella: report of 30 cases. Urol Int, 1992;48(4):395-400.

5. Fawzia E, Al-Otaibi. Isolation of Salmonella paratyphi A from a patient with nephrolithiases. Saudi Med J, 2003;24(4):406-408. 